Research Article

\title{
Knowledge Service Technology for Supporting Intelligent Product Design
}

\author{
Haiqiao Wang (i) and Ruikun Niu \\ School of Mechanical and Electrical Engineering, Jinling Institute of Technology, Nanjing, Jiangsu, China \\ Correspondence should be addressed to Haiqiao Wang; wanghaiqiao@jit.edu.cn
}

Received 13 July 2021; Revised 21 September 2021; Accepted 1 November 2021; Published 18 November 2021

Academic Editor: Bin Niu

Copyright (c) 2021 Haiqiao Wang and Ruikun Niu. This is an open access article distributed under the Creative Commons Attribution License, which permits unrestricted use, distribution, and reproduction in any medium, provided the original work is properly cited.

\begin{abstract}
In this paper, a knowledge service method that supports the intelligent design of products is investigated. The proposed method provides the solutions to computational problems and reasoning and decision-making problems in the field of intelligent design. The requirement analysis of a knowledge-based intelligent design system integrates design knowledge into case-based reasoning activities through scheme analysis, scheme evaluation, and scheme adjustment, thus achieving knowledge-based intelligent reasoning and decision-making. During the similarity matching, a new hybrid similarity measurement method is proposed to calculate the similarity of crisp and fuzzy sets. This method integrates the fuzzy set similarity theory based on the traditional similarity measurement method. A method of attribute level classification is proposed to assign weight coefficients. The attributes are divided into the primary matching and auxiliary matching levels according to the decisiveness of case matching, and the set of weight coefficients is continuously and dynamically updated through case-based reasoning learning. Then, the weighted global similarity measure is used to obtain the set of similar cases from the case database. Finally, a design example of a computer numerical control tool holder product is studied to present the practicability and effectiveness of the proposed method.
\end{abstract}

\section{Introduction}

Currently, the increasingly competitive market environment has been forcing enterprises to strengthen their product development and their ability to respond quickly to market demands. The intelligent design methods can simultaneously meet the needs of rapid and automated product development, respond to the current needs of market diversification and customization, avoid many repetitions, shorten the research and development cycle, and enhance the competitiveness of products [1]. Therefore, they have been highly valued by researchers and enterprises. Product design is a knowledge-driven innovative process that includes analysis, synthesis, and decision-making, while the intelligent design of products represents a result of the application of intelligent engineering and decision-making automation technology in the design field. The main goal of intelligent design is to solve two types of design problems: computational problems and reasoning and decision-making problems.
The computer numerical control (CNC) tool holder is the core functional component of CNC machine tools with a wide variety of variable working conditions, diverse configuration requirements, and small market demand for a single product. However, its intelligent design is a knowledge-intensive and complex process. The structural design of the $\mathrm{CNC}$ tool holder products is a process of inheriting and reusing the design knowledge. Commonly, the typical design schemes that had been successfully developed and design knowledge and experience have been used in the design process. In the traditional design process of the $\mathrm{CNC}$ tool holder products, the design has to be performed for various performance indicators, and the design scheme of a CNC tool holder cannot be completed without many tests and verifications. The entire design process not only is timeconsuming, but also greatly relies on a designer's experience and expertise. Intelligent product design plays an important role in product design because it can achieve the inheritance, can reuse the product design process knowledge, and can reduce unnecessary repetitive design activities [2]. 
The basis for achieving an intelligent product design is a structured description and processing of design knowledge, which are necessary to realize the function of computer reasoning. To address the lack of semantic understanding of the existing injection mold intelligent design systems based on case-based reasoning (CBR), Guo et al. [3] introduced the ontology technology into the description of cases to achieve their semantic retrieval, which improved the system's recognition of the designer's design intent in the conceptual design phase. Zhang et al. [4] proposed a knowledge push technology based on applicable probabilistic matching and multidimensional context driving and constructed a training sample set, which includes knowledge description vectors, case feature vectors, and Boolean mapping matrixes, to describe the matching degree between knowledge and content. Khosravani and Nasiri [5] proposed case-based reasoning and applied it to the injection molding process. The injection molding CBR system was used for process design, process parameters design, fault diagnosis, and enhanced quality control. Wei et al. [6] proposed an intelligent design method for automobile interior structure based on rule-based reasoning. The method was designed as a three-layer architecture, including the interaction, reasoning, execution, and knowledge base layers. The intelligent design of the interior decoration structure of a car can be achieved through rule-based reasoning. The existing research has improved knowledge-based intelligent design to a certain extent, but it still lacks a method that can provide a solution to the entire process of intelligent product design, which affects a comprehensive solution to the computational problems and reasoning and decision-making problems of intelligent design systems.

Many similarity calculation investigations and research on multiattribute decision-making can be found in the literature, as shown in Table 1.

In summary, to improve the retrieval efficiency in similar cases further, the above-mentioned studies have made improvements in similarity calculation, feature weight coefficient allocation, and case library optimization. For instance, the similarity calculation has been improved by methods such as hesitant fuzzy hybrid vector and weighted heterogeneous value distance measurement, genetic algorithm, particle swarm algorithm, and multistandard decisionmaking; the iterative weight update was used in the calculation of feature weight coefficients, thus avoiding excessive reliance on subjective judgments and making the retrieval more efficient. Although these methods have improved the retrieval efficiency and accuracy, there is still space for further improvement in the intelligent design of CNC tool holder products. The conceptual design of a CNC tool holder includes five attribute value formats, but there has been no unified calculation formula for the fuzzy attribute sets yet. In addition, a long time is needed for the existing retrieval schemes to find an optimal solution. Furthermore, the coefficients of the attribute weight set should be continuously and dynamically updated with the update of the case database.

To overcome the aforementioned challenges, a novel knowledge service method that supports the intelligent design of products is proposed in this paper. Based on the design knowledge of the previous product design standards and expert experience and combined with the case-based reasoning and rule-based reasoning mechanisms, the proposed method can effectively solve both computational problems and reasoning decision-making problems in intelligent product design. The contributions of this paper can be summarized as follows.

Through a detailed analysis of the existing research results of the comparison of the similarity measurement methods, the shortcomings of the existing methods for case retrieval of CNC tool holders are identified. Most of the existing methods for hybrid similarity measurement consider two, three, or four attribute values formats. Furthermore, based on the CNC tool holder product instances, the product attributes can be divided into five formats: Crisp Symbols (CS), Crisp Numbers (CN), Fuzzy Numbers (FN), Fuzzy Linguistic variables (FL), and Fuzzy Intervals (FI). The proposed method comprehensively considers multiple formats of attribute values involved in the process of case retrieval. These formats basically cover all types of attribute values in practical CBR applications. A similarity calculation equation is developed for fuzzy attribute sets. A novel hybrid similarity calculation method is proposed to consider the similarity between the crisp and fuzzy sets comprehensively.

In the design of mechanical products, such as CNC tool holder products, the design resource library is a fundamental factor. In the conceptual design, the effects of various performance attributes of the CNC tool holder products on the case retrieval are considered. This is based on factors such as the design standards, experience rules, and professional domain knowledge in the design resource library. A method of the attribute level classification to assign weight coefficients is proposed, and attributes are divided into primary and auxiliary matching levels according to the decisiveness of instance matching. Then, the tool holder model, tool holder configuration, and power source are taken as a primary matching level. As long as one of the three attributes does not match, the final similarity will be zero, which can reduce the time to find an optimal solution. Furthermore, in most of the existing studies, the weight coefficient will not change once it is determined. In this paper, the weight coefficient can be continuously and dynamically updated through case-based reasoning learning. Finally, through a design case of a CNC tool holder product, the method's ability to solve the reasoning and decisionmaking design problems is verified.

The rest of this paper is structured as follows. In Section 2 , the general idea of knowledge service supporting the intelligent design of products is proposed. In Section 3, a new hybrid similarity measurement method with five attribute value formats in scheme reasoning is proposed; also, a similarity calculation equation is derived for fuzzy attribute sets, and a method for attribute level classification is proposed to assign weight coefficients. In Section 4, an automatic calculation of computational problems in the intelligent design process is verified through a design case of a CNC tool holder product. The conclusions are presented in Section 5 . 
TABLE 1: Related research literature.

Related research literature

Zhang and $\mathrm{Yu}$ [7] proposed two distance measures of intuitionistic and interval-valued fuzzy sets. Sharaf-El-Deen et al. [8] proposed a new hybrid case-based reasoning approach for medical diagnosis systems. Qian et al. [9] proposed an improved requirements-driven self-adaptation approach that combines goal reasoning and casebased reasoning. Gu et al. [10] proposed a CBR system based on the weighted heterogeneous value distance metric. Tan et al. [11] described the hesitant fuzzy Hamacher aggregation operators for multicriteria decision-making. Song et al. [12] proposed a general type of similarity measure for an intuitionistic fuzzy set with two parameters. Mahmood et al. [13] proposed the notion of complex hesitant fuzzy hybrid vector similarity measures. Feng and Khan et al. $[14,15]$ proposed a case library optimization method to improve detection efficiency.

Feng et al. [16] combined the genetic algorithm and the particle swarm optimization algorithm in the calculation of attribute feature weights. Xiong et al. [17] have concluded that the normalization factor directly determines the influence of the corresponding attributes on the filtering result. Ahmadvand and Pishvaee [18] proposed a credibility-based fuzzy common weights data envelopment analysis approach. Li et al. [19] developed an integrated cumulative prospect theory based on the hybridinformation multicriteria decision-making approach. Lin et al. [20] reported that the traditional TODIM method could be extended to handle the HFLTSs based on the novel comparison function and distance measure. Chen et al. [21] proposed a two-stage logic scoring of the preference-ELECTRE III-based approach. Sun et al. [22] proposed an iterative weight update method based on the optimistic coefficient.

\section{General Idea of Knowledge Service Supporting the Intelligent Design of Products}

\subsection{Requirement Analysis of Intelligent Product Design.} An intelligent design is a modern design method that combines knowledge-based engineering (KBE) and computer-aided design theory. This method aims to enable the design system to use the abundant knowledge, such as expert experience, design manuals, standards, and industry norms, to reason, judge, make decisions, and achieve customization, automation, and intelligence of product design by simulating the cognitive and the behavioral characteristics of designers in the design activities [23-25].

The product design process is essentially an iterative decision-making process that includes user requirement assessment, design process decision-making, technical problem decision-making, and evaluative decision-making. The completion of these tasks requires using computeraided design tools, as well as a large amount of knowledge for reasoning, judgment, and decision-making [26]. The product design process requires the development of the intelligent design to a higher level of automation to support large-scale multidisciplinary and multidomain knowledge integration. The purpose of supporting intelligent product design is to replace the decision-making and reasoning processes of experts and designers with the KBE and artificial intelligence technologies. Moreover, its purpose is to intelligently achieve product design and knowledge reuse by
Method advantages and disadvantages

The existing research on similarity calculation investigations has improved the accuracy of case retrieval and has made the retrieval process more reasonable. The proposed methods have the following advantages: (1) they enhance the ability of a CBR system to process nonlinear data; (2) they overcome the problem of difficulty in obtaining decision-making knowledge in cases of discontinuous information; (3) they can handle continuous and discrete attributes simultaneously.

However, some of the methods have certain limitations, which are as follows: (1) the classification of attribute types is not detailed enough; (2) the research on the similarity calculation of fuzzy attribute sets is not deep enough.

The existing research on multiattribute decision-making not only can improve the matching accuracy but can also avoid excessive reliance on decision-makers to set the weight value subjectively. The proposed methods have the following advantages: (1) they consider the psychological behavior of decision-makers; (2) the reasoning logic pattern in the human cognition process and the compensation among attributes are taken into account; (3) multiple methods are included to deal with multiattribute decision-making problems.

However, some of these methods have certain limitations: (1) it takes a long time to find an optimal solution; (2) the coefficients of the attribute weight set are not continuously and dynamically updated.

constructing a design process-oriented knowledge environment, thus reducing the cycle iteration time and repetitive work in the product design process $[27,28]$.

The knowledge service supporting the intelligent design of products is a human-computer interaction design activity that integrates multidomain knowledge and employs computer-aided design tools $[29,30]$. To clarify the functional goals of the knowledge-based intelligent design system, it is necessary to analyze the demand for intelligence in the actual product design process according to the characteristics of product design. The functional goals are as follows:

(1) Support integrated management of distributed and heterogeneous knowledge. The product design knowledge has the characteristics of distribution, intensiveness, and heterogeneity. Most of the current product data management systems provide partial document data classification and storage and generally have only the keyword-based retrieval function. This is less effective for knowledge integration and service and is not conducive to the reuse of knowledge by designers. Therefore, the intelligent design system first needs to realize the integrated management of distributed and heterogeneous knowledge.

(2) Support the design tool integration and process encapsulation. The competition of design tasks 
usually requires the collaborative use of multiple types of design tools. For instance, during the design of CNC tool holders, the Unigraphics (UG) software has been typically used for three-dimensional modeling, while MATLAB, ANSYS, and other software have been used for design activities such as calculation and simulation. This is a process of iterations and continuous corrections. It requires the intelligent design system to integrate different design tools and reuse the design process to support an iterative solution to the computational problems.

(3) Support intelligent reasoning and decision-making. The intelligence of the intelligent product design is reflected in the ability of reasoning and decisionmaking similar to human thinking during the design process. It requires computers to achieve automatic processing and auxiliary/automatic decision support for multidomain and multitype design knowledge.

2.2. Knowledge Service Method for Intelligent Product Design. According to the requirements of the intelligent design process, the content of knowledge service supporting the intelligent design of products can be divided into the following three parts: the integration and management of distributed and heterogeneous knowledge, the integration of design tools and encapsulation process, and the reasoning of design knowledge and auxiliary decision-making. The integration and management of distributed and heterogeneous knowledge mainly provide the service-oriented knowledge reuse for the intelligent reasoning of design knowledge and auxiliary decision-making to solve the reasoning decisionmaking problems. Based on these two parts, the integration of design tools and encapsulation process solves the computational problems based on the knowledge-based parametric drive and regularization constraints. These three parts are independent and interrelated and jointly provide a solution to the computational problems and reasoning and decision-making problems in intelligent product design. The characteristics of intelligent design are analyzed, and a knowledge-based intelligent design system is constructed according to the relationship between the design process and design knowledge. The overview of the intelligent product design knowledge service system is shown in Figure 1.

\section{Key Technologies of Knowledge-Based Intelligent Product Design System}

3.1. Description of Product Design Problems of CNC Tool Holders. A CNC tool holder is a key component of a CNC machine tool. After investigating the research and development of a CNC tool holder, the design contents of the $\mathrm{CNC}$ tool holder can be categorized as structural design, precision design of the indexing mechanism, strength design, and calibration of the transmission mechanism, as shown in Figure 2. A large amount of design knowledge, including design manuals, standard specifications, empirical rules, and successful cases, is required in the design process of CNC tool holders to accommodate different types of CNC machine tools and to achieve good running accuracy, indexing accuracy, and part strength.

The conceptual design attributes of a CNC tool holder are extracted based on the index parameters of user performance requirements. Conceptual design cases of the CNC tool holder are expressed according to the attributes of the tool holder model, structural characteristics, accuracy characteristics, power indicators, and power sources, as shown in Table 2. The structural characteristics include the geometrical and structural characteristics of the CNC tool holder. The accuracy characteristics represent the accuracy evaluation index of the user requirements of the CNC tool holder. The performance characteristics mainly include the dynamic performance indicators of a servo tool holder. These attributes can be divided into five data types: Crisp Symbols (CS), Crisp Numbers (CN), Fuzzy Numbers (FN), Fuzzy Linguistic variables (FL), and Fuzzy Intervals (FI). These types include crisp and fuzzy sets [31]. The parameters of these attributes are used to retrieve similar design cases in the case library that are then used as design prototypes. These design prototypes are adjusted and modified to meet the user's requirements in the detailed design phase.

\subsection{Scheme Reasoning and Auxiliary Decision-Making.}

The CBR technology is a form of artificial intelligence used for the development of simple machine imitation to deep machine thinking. However, the existing CBR research has certain shortcomings in the scheme evaluation and parameter modification. Therefore, in this study, the related knowledge involved in the design activities is integrated into the reasoning engine by constructing a knowledge environment that can play an auxiliary decision-making role $[32,33]$. As shown in Figure 3, the scheme reasoning and decision-making process can be divided into scheme analysis, scheme evaluation, and scheme adjustment. The main design activities include the reasoning of design cases and the auxiliary decision-making of design knowledge.

A set of design cases similar to the target solution can be obtained by the reasoning engine through case retrieval. The existing CBR-based intelligent design methods select the case with the highest degree of similarity as a target solution [34-36]. However, during the actual design process, similar matched cases will play only a partial role in a new design. In other words, the feature attributes of an object used for case matching cannot completely determine the reusability of a particular case, and the evaluation of a solution depends on various evaluation indexes, such as failure rate, structural simplicity, maintainability, safety, and economic cost. Therefore, the system proposed in this paper adopts a scheme evaluation method that combines the gray correlation analysis and the entropy method to quantify the relationship between the evaluation index and scheme. Finally, a more scientific and reasonable similar optimal case is selected as a target scheme. After obtaining a similar optimal scheme, the system verifies the target scheme through the mapping relationship between the design requirements index and design parameters. For parameters that do not meet the design requirements, further modification is 


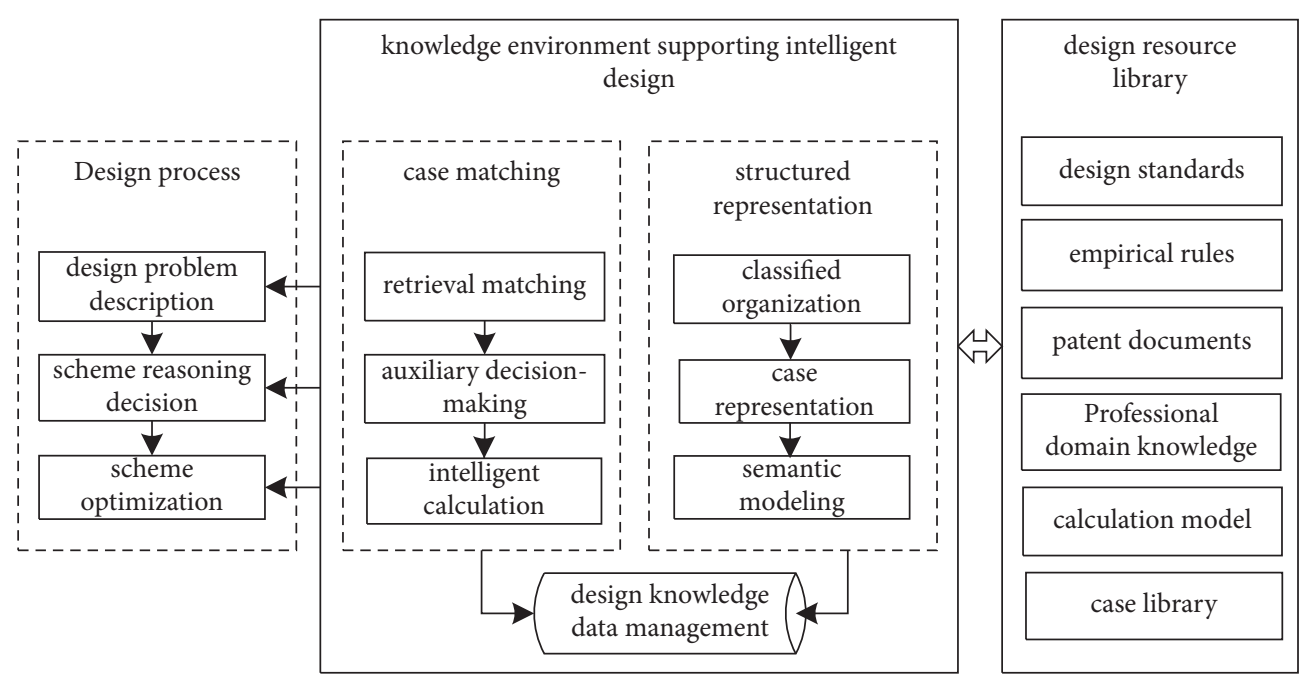

Figure 1: The overview of the intelligent product design knowledge service system.

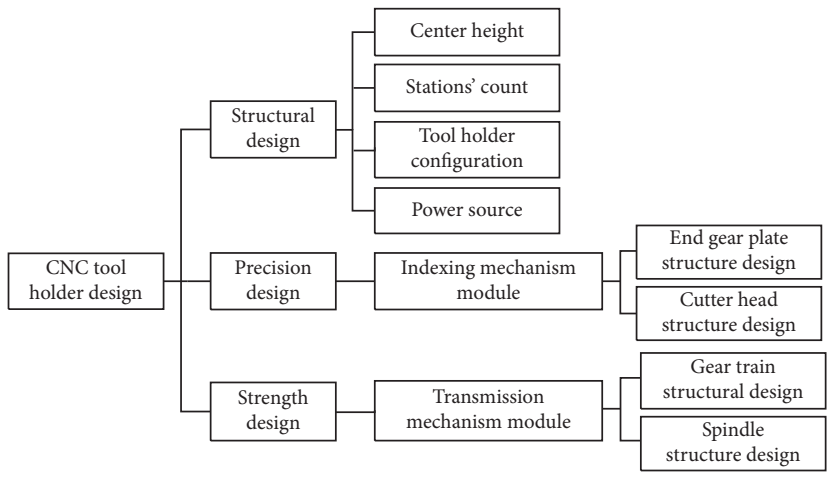

FIgURE 2: Design content diagram of the CNC tool holders.

TABle 2: Product performance requirements of the CNC tool holders.

\begin{tabular}{lccc}
\hline No. & Attribute & Index & Data type \\
\hline 1 & Model & Tool holder model & CS \\
\hline 2 & & Center height $(\mathrm{mm})$ & CN \\
3 & Structure & Stations count $(\mathrm{N})$ & CN \\
4 & & Net weight (without cutter head) & FL \\
5 & Tool holder configuration (front/rear) & FN \\
\hline 6 & Accuracy & Turn $30^{\circ}$ and lock $(\mathrm{S})$ & FN \\
7 & & Turn $180^{\circ}$ and lock $(\mathrm{S})$ & FI \\
8 & & Repeat positioning accuracy $(\mathrm{mm})$ & $\mathrm{CN}$ \\
9 & Performance & Maximum unbalanced moment $(\mathrm{Nm})$ & $\mathrm{CN}$ \\
10 & & Maximum tangential moment $(\mathrm{kNm})$ & $\mathrm{CN}$ \\
11 & & Maximum axial torque $(\mathrm{kNm})$ & \\
\hline 13 & Power source & Maximum load $(\mathrm{kg})$ & \\
\hline
\end{tabular}

required to obtain the final design scheme, which is then saved to the database for purposes of sharing and reuse.

3.3. Case Similarity Matching Algorithm. Case retrieval is a process of quickly matching similar cases in a case database according to the user requirements. The key technology is to establish an efficient case retrieval mechanism. The index attributes of the performance requirement of the CNC tool holder product are classified in Table 1. According to the new design problem requirements, a designer describes and classifies product attributes first and then matches similar 


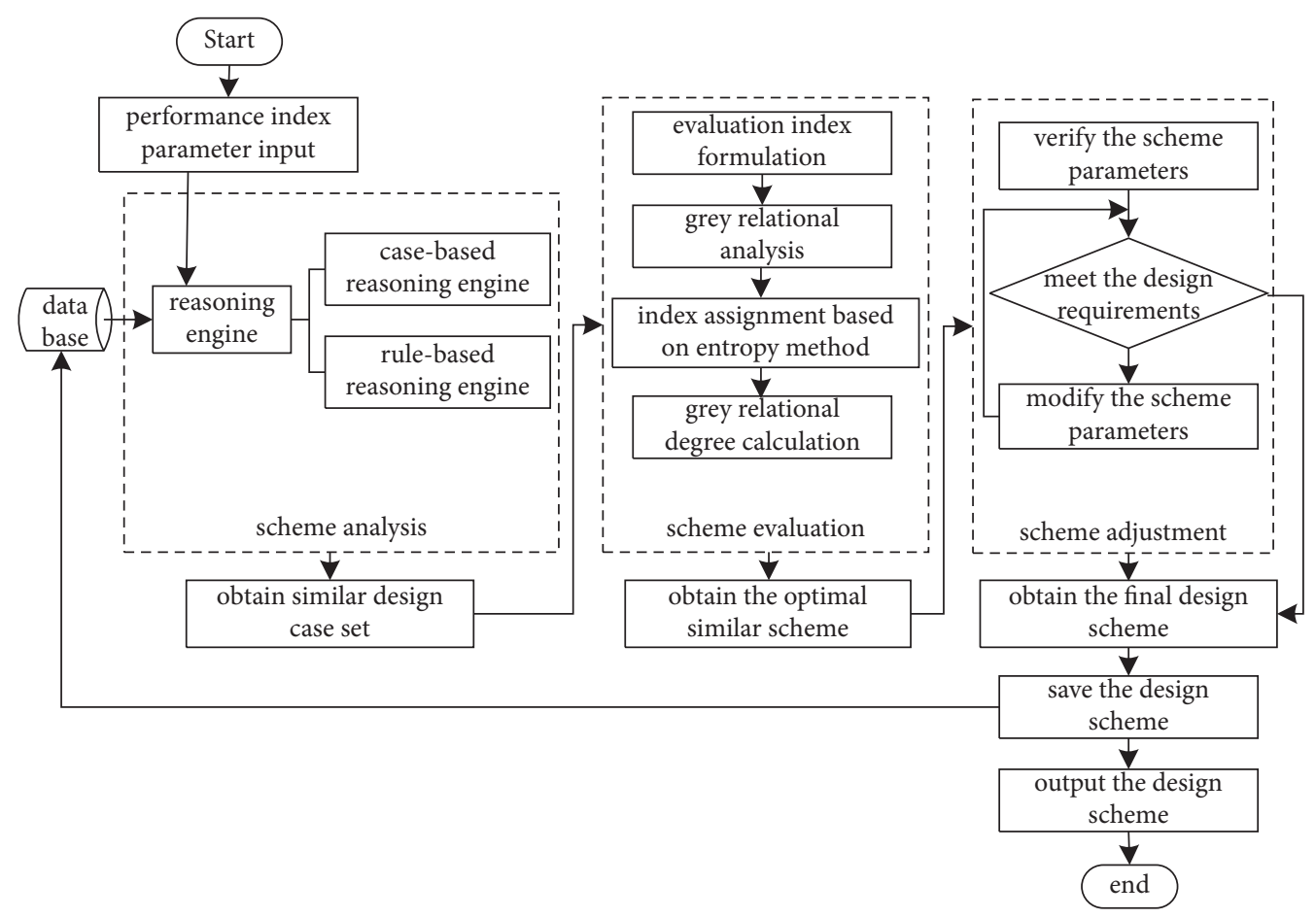

FIgURE 3: Scheme reasoning and decision-making process.

cases through similarity metrics. Most of the similarity measurement methods proposed in the CBR field consider the matching of crisp set attributes, while in-depth research on the similarity matching of fuzzy set attributes has been inadequate $[37,38]$. In the case of similarity measurement, the fuzzy set similarity theory can naturally simulate the actual situation [39]. Therefore, a hybrid similarity measurement method that synthesizes both crisp and fuzzy sets of the CNC tool holder products is proposed in this study to ensure the efficiency of the CBR system retrieval mechanism.

Typically, the CBR system uses the Euclidean or Hamming distance as a similarity metric of the crisp set, which can be expressed as

$$
\operatorname{sim}\left(x_{i}, y_{i}\right)=1-\operatorname{dist}\left(x_{i}, y_{i}\right)
$$

where $x_{i}$ and $y_{i}$ denote the $i$ th attribute value of the two cases, while $\operatorname{sim}\left(x_{i}, y_{i}\right)$ and $\operatorname{dist}\left(x_{i}, y_{i}\right)$ are the similarity value and the distance between the $i$ th attribute in the two cases, respectively. For the $\mathrm{CN}$ attribute value, $\operatorname{dist}\left(x_{i}, y_{i}\right)=\left|x_{i}-y_{i}\right| /\left(\max _{i}-\min _{i}\right)$, where $\max _{i}$ and $\min _{i}$ are the upper and lower limits of the $i$ th attribute value, respectively. For the CS attribute value, when $x_{i}=y_{i}$, then $\operatorname{dist}\left(x_{i}, y_{i}\right)=0$; otherwise, $\operatorname{dist}\left(x_{i}, y_{i}\right)=1$.

The similarity measure of the crisp set is no longer applicable to the fuzzy set. Therefore, in this study, the relative area method is used to calculate the similarity between two fuzzy sets as follows:

$$
\begin{aligned}
\operatorname{sim}\left(x_{i}, y_{i}\right) & =\frac{S\left(x_{i} \cap y_{i}\right)}{S\left(x_{i} \cap y_{i}\right)} \\
& =\frac{S\left(x_{i} \cap y_{i}\right)}{S\left(x_{i}\right)+S\left(y_{i}\right)-S\left(x_{i} \cap y_{i}\right)},
\end{aligned}
$$

where $S$ represents the area of the corresponding membership function; $x_{i}$ and $y_{i}$ represent the $i$ th attribute values of the two cases in the similarity metric; $x_{i} \cap y_{i}$ and $x_{i} \cup y_{i}$ represent the intersection and the union of the two fuzzy attribute domains, respectively. Four types of intersections of two fuzzy attribute domains are shown in Figure 4.

In Figure 4(d), $S\left(x_{i} \cap y_{i}\right)$ remains unchanged since the two attribute sets are inclusive. According to equation (2), the change in the center distance between the two fuzzy attribute domains does not affect their similarity. However, this does not conform to the similarity calculation of the fuzzy attribute type FL. Based on the above analysis, the fuzzy attribute domain similarity measurement methods developed for these four types are as follows:

$c_{x i}=(\underline{\alpha}+\bar{\alpha}) / 2, \quad c_{y i}=(\beta+\bar{\beta}) / 2 / /$ calculate the midpoint $c_{x i}$ and $c_{y i}$ of the fuzzy set; $\underline{\alpha}(\beta)$ and $\bar{\alpha}(\bar{\beta})$ denote the upper and the lower limits of the fuzzy attribute domain, respectively.

If $c_{x i}>c_{y i}$, then exchange $x_{i}$ and $y_{i}$, endif.

$a=0.1 \underline{\alpha}, b=0.1 \bar{\alpha}, c=0.1 \beta, d=0.1 \bar{\beta}$.

$x_{i}^{*}=(b \underline{\beta}+c \bar{\alpha}) /(c+b), y_{i}^{*}=1-\left(x_{i}^{*}-\bar{\alpha}\right) / b / /$ calculate the intersection $\left(x_{i}^{*}, y_{i}^{*}\right)$.

If $y_{i}{ }^{*} \leq 0$, then $\mathrm{S}\left(x_{i} \cap y_{i}\right) \leftarrow 0, \operatorname{sim}\left(x_{i}, y_{i}\right) \leftarrow 0$ //belongs to type (a).

Else $\left.S\left(x_{i}\right) \leftarrow(2 \bar{\alpha}+b-2 \underline{\alpha}+a) / 2\right), \quad S\left(y_{i}\right) \leftarrow(2 \bar{\beta}+d-$ $2 \beta+c) / 2 / /$ calculate $S\left(x_{i}\right)$ and $S\left(y_{i}\right)$.

If $0<y_{i}^{*}<1$, then $S\left(x_{i} \cap y_{i}\right) \leftarrow(\bar{\alpha}+b-\beta+c) y_{i}^{*} / 2$ //belongs to type (b).

Else//belongs to type (c) or (d). 


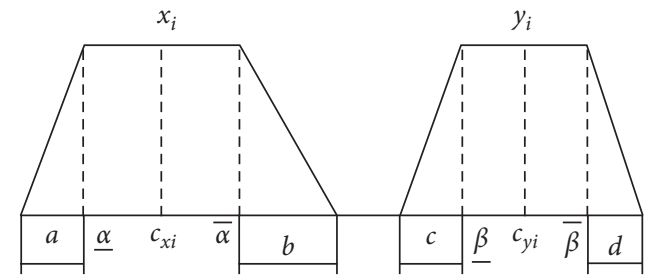

(a)

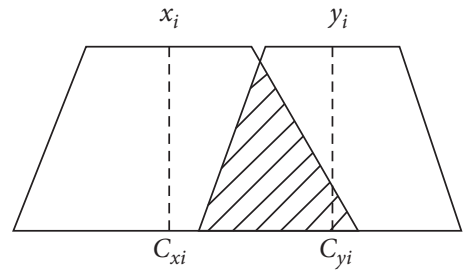

(b)

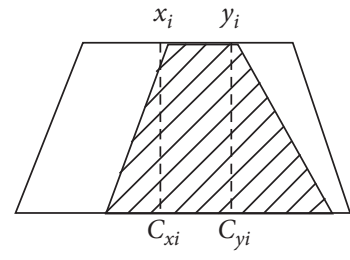

(c)

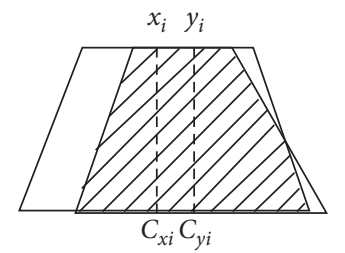

(d)

FIgURE 4: The intersection types between two fuzzy attribute domains $x_{i}$ and $y_{i}$. (a) Type (a), (b) type (b), (c) type (c), and (d) type (d).

If $\bar{\alpha}+b<\bar{\beta}+d$ and $\underline{\alpha}-a<\beta-c$, then $S\left(x_{i} \cap y_{i}\right) \leftarrow(2 \bar{\alpha}+$ $b-2 \beta+c) / 2 / /$ belongs to type (c). (d).

Else $S\left(x_{i} \cap y_{i}\right) \leftarrow \min \left(S\left(x_{i}\right), S\left(y_{i}\right)\right)$ //belongs to type

endif.

endif.

$\operatorname{sim}\left(x_{i}, y_{i}\right) \leftarrow S S\left(x_{i} \cap y_{i}\right) /\left(S\left(x_{i}\right)+S\right.$

$\left.\left(y_{i}\right)-S\left(x_{i} \cap y_{i}\right)\right)$.

endif.

3.4. Global Similarity Measurement. The attribute types of $\mathrm{CNC}$ tool holder products include crisp and fuzzy sets. Thus, a hybrid similarity metric should be employed to calculate the similarity between the attributes $[40,41]$. Another important aspect of improving the performance of similarity measurement is to assign reasonable attribute weight coefficients according to the relative importance of attributes $[31,42]$. The attributes can be classified into two levels according to the analysis of the product structure, accuracy, and performance indicators, as well as the importance and decisiveness of the product attribute for case matching. The first level is for primary matching and the second level is for auxiliary matching.

No weights are assigned for attributes belonging to the primary matching level, and the similarity measurement equation is expressed as follows:

$$
\begin{aligned}
\operatorname{sim}_{1}(X, Y)= & \operatorname{sim}_{\mathrm{CS}}\left(x_{i}, y_{j}\right) \times \operatorname{sim}_{\mathrm{CN}}\left(x_{j}, y_{j}\right) \\
& \times \operatorname{sim}_{\mathrm{FNI}}\left(x_{k}, y_{k}\right) \times \operatorname{sim}_{\mathrm{FL}}\left(x_{l}, y_{l}\right) .
\end{aligned}
$$

For the auxiliary matching level, the weighted average operator is used to aggregate the similarity measures between case attributes to obtain the level similarity. The weight coefficients are assigned by binary comparison evaluation and normalization of the weight set performed by multiple experts. Moreover, the weight set is stored in the system as a reference. The weight set is not static, and during the similarity measurement retrieval, the CBR system can continuously modify the weight set through learning to make it closer to the actual situation of the product design [43]. The similarity measurement equation for the auxiliary matching level is as follows:

$$
\begin{aligned}
\operatorname{sim}_{2}(X, Y)= & \sum_{i} w_{i} \operatorname{sim}_{\mathrm{CS}}\left(x_{i}, y_{i}\right) \\
& +\sum_{j} w_{j} \operatorname{sim}_{\mathrm{CN}}\left(x_{j}, y_{j}\right)+\sum_{k} w_{k} \operatorname{sim}_{\mathrm{FNI}}\left(x_{k}, y_{k}\right) \\
& +\sum_{l} w_{l} \operatorname{sim}_{\mathrm{FL}}\left(x_{l}, y_{l}\right) .
\end{aligned}
$$

In equations (3) and (4), $X$ and $Y$ represent two instances; $\operatorname{sim}_{\mathrm{CS}}\left(x_{i}, y_{i}\right), \operatorname{sim}_{\mathrm{CN}}\left(x_{j}, y_{j}\right)$, and $\operatorname{sim}_{\mathrm{FL}}\left(x_{l}, y_{l}\right)$ are the similarity values of the attribute categories CS, CN, and $\mathrm{FL}$, respectively; $\operatorname{sim}_{\mathrm{FNI}}\left(x_{k}, y_{k}\right)$ is the similarity value between the FN and FI attributes; $w_{i}, w_{j}$, and $w_{l}$ are the weight coefficients of the attribute categories CS, CN, and FL in the matching level, respectively; $w_{k}$ is the weight coefficient of the FN and FI attributes, and $\sum w_{i}+\sum w_{j}+\sum w_{k}+\sum w_{l}=1$ ; parameters $i, j$, and $l$ are the numbers of attributes of the CS, $\mathrm{CN}$, and FL categories of the two matching levels, respectively; $k$ is the total number of attributes of FN and FI categories.

For the similarity measurement of attribute types FN and FI, it is necessary to consider the influence of the center distance of the two fuzzy attribute domains on the similarity measurement. The calculation equation is expressed as

$$
\operatorname{sim}_{\mathrm{FNI}}\left(x_{k}, y_{k}\right)=\varepsilon_{k 1} \operatorname{sim}_{1_{\mathrm{FNI}}}\left(x_{k}, y_{k}\right)+\varepsilon_{k 2} \operatorname{sim}_{2_{\mathrm{FNI}}}\left(x_{k}, y_{k}\right),
$$

where $\operatorname{sim}_{\mathrm{FNI}}\left(x_{k}, y_{k}\right)$ is the similarity measurement equation of attribute types FN and FI; $\varepsilon_{k 1}$ and $\varepsilon_{k 2}$ are the coefficients of $\operatorname{sim}_{1_{\mathrm{FNI}}}\left(x_{k}, y_{k}\right)$ and $\operatorname{sim}_{2_{\mathrm{FNI}}}\left(x_{k}, y_{k}\right)$, respectively, which are set by experts, stored in the retrieval system, and dynamically updated with the inclusion of new cases into the case 


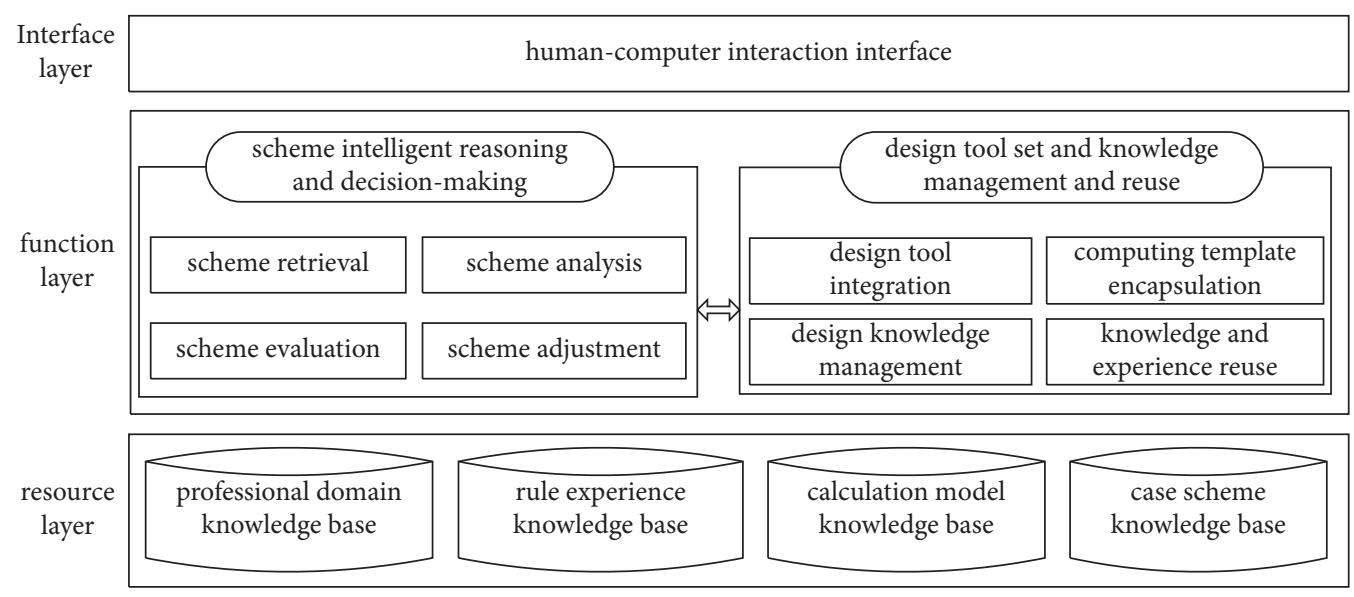

Figure 5: Technical system structure of the intelligent product design.

database; $\operatorname{sim}_{1_{\mathrm{FNI}}}\left(x_{k}, y_{k}\right)$ is obtained from the fuzzy set similarity by equation $(2) ; \operatorname{sim}_{2_{\mathrm{FNI}}}\left(x_{k}, y_{k}\right)$ represents the influence of the distance between the centers of two fuzzy sets on the similarity and it is calculated by $\operatorname{sim}_{2_{\mathrm{FNI}}}\left(x_{k}, y_{k}\right)=1-\operatorname{dist}\left(c_{x k}-c_{y k}\right)=1-\left|c_{x k}-c_{y k}\right| / \max _{k}$.

Based on the hybrid similarity measurement method and the classification of matching weight coefficients, the global similarity of design case feature attribute matching in case retrieval can be expressed as follows:

$$
\operatorname{sim}(X, Y)=\operatorname{sim}_{1}(X, Y) \times \operatorname{sim}_{2}(X, Y) .
$$

\section{Verification through Application Examples}

4.1. Intelligent Design System Structure. The architecture of the knowledge-based intelligent design can be divided into three layers: the interface layer, the function layer, and the resource layer, as shown in Figure 5. The interface layer has a friendly human-computer interaction interface that enables users to communicate with the system during operation and guides users to complete the design process of CNC tool holder products. The functional layer includes intelligent reasoning and decision-making of the scheme, design toolset, knowledge management, and reuse. This can help in effectively integrating the knowledge module with other modules and driving the product design by knowledge. The resource layer is mainly composed of the professional domain knowledge base, rule experience knowledge base, calculation model knowledge base, and case scheme knowledge base.

4.2. Application Examples. Before scheme reasoning in the intelligent design system, the designers are first required to set the matching level and weighting coefficients for each performance index of the CNC tool holder product according to expert opinions. Weight matching levels and the assigned weighting coefficients of the corresponding performance index are shown in Table 3. The system automatically executes the case matching function according to the similarity calculation method corresponding to different performance index attribute types. Then, the system obtains a set of similar cases.

$$
\operatorname{sim}(X, Y) \geq T
$$

Taking the product design of the CNC tool holder as an example, its product attribute types include crisp and fuzzy sets. Thus, the global similarity measurement method is applied to the design of the CNC tool holder product based on case-based reasoning. Suppose that $X\left(X=\left(x_{1}, x_{2}, \ldots, x_{13}\right)\right)$ and $Y\left(Y=\left(y_{1}, y_{2}, \ldots, y_{13}\right)\right)$ are the new design case and the case in the case library of the CNC tool holder design, respectively, where $x_{i}$ and $y_{i}$ correspond to the attributes of $X$ and $Y$, respectively, as shown in Table 2. In terms of weight coefficient assignment, no weight coefficient is assigned for the primary matching level. The weight coefficient of each attribute is given by the weight set in the system for the auxiliary matching level. The attribute data types, matching levels, weight coefficients, attribute values, and global similarity measurements of each attribute for new and old cases are given in Table 3 .

According to Table 3, the global similarity value between the new design case and a case in the case library is 0.632 . In equation (7), $T$ represents the similarity threshold of the retrieved cases. All similar sets of cases whose similarity with the new design case is greater than the given threshold can be retrieved in the case library. The designers configure the weight of each evaluation index of the CNC tool holder design scheme determined by experts in the professional field, such as failure rate, structural simplicity, maintainability, safety, and economic cost. The system automatically calculates the gray correlation degree of each similar scheme according to the weight of each index. Then, it sorts and obtains the design scheme with the largest gray correlation degree as an optimal similarity case. The intelligent design system automatically verifies the specific design scheme corresponding to the similar optimal case, checks whether each parameter in the scheme meets the requirements, and automatically modifies the scheme parameters based on the relevant knowledge from the rule experience knowledge base to obtain the final design scheme and saves it to the database. 
TABLE 3: Similarity measurement of the performance requirements of the CNC tool holder.

\begin{tabular}{|c|c|c|c|c|c|c|}
\hline Index & $\begin{array}{l}\text { Data } \\
\text { type }\end{array}$ & $\begin{array}{c}\text { Matching } \\
\text { level }\end{array}$ & $\begin{array}{l}\text { Weight } \\
\text { coefficient }\end{array}$ & New case $\mathrm{X}$ & Case $\mathrm{Y}$ in the library & Global similarity \\
\hline Tool holder model & CS & \multirow{3}{*}{ Primary } & \multirow{3}{*}{ - } & \multirow{3}{*}{$\begin{array}{c}\text { SLT } \\
\text { Front } \\
\text { Servo } \\
\text { motor }\end{array}$} & SLT & 1 \\
\hline Tool holder configuration & CS & & & & Front & 1 \\
\hline Power source & CS & & & & Servo motor & 1 \\
\hline Center height $(\mathrm{mm})$ & $\mathrm{CN}$ & \multirow{10}{*}{ Auxiliary } & 0.12 & 80 & 125 & 0.084 \\
\hline Stations' count $(\mathrm{N})$ & $\mathrm{CN}$ & & 0.12 & 8 & 12 & 0.080 \\
\hline Net weight (without cutter head) & FL & & 0.06 & Light & Medium & 0.003 \\
\hline Turn $30^{\circ}$ and lock (S) & FN & & 0.11 & 0.3 & 0.41 & 0.049 \\
\hline Turn $180^{\circ}$ and lock (S) & $\mathrm{FN}$ & & 0.11 & 0.6 & 0.86 & 0.052 \\
\hline Repeat positioning accuracy $(\mathrm{mm})$ & FI & & 0.12 & $<0.003$ & $<0.003$ & 0.120 \\
\hline $\begin{array}{l}\text { Maximum unbalanced moment } \\
(\mathrm{Nm})\end{array}$ & $\mathrm{CN}$ & & 0.1 & 30 & 50 & 0.080 \\
\hline $\begin{array}{l}\text { Maximum tangential moment } \\
(\mathrm{kNm})\end{array}$ & $\mathrm{CN}$ & & 0.1 & 1.51 & 3.6 & 0.079 \\
\hline Maximum axial torque $(\mathrm{kNm})$ & $\mathrm{CN}$ & & 0.1 & 1.62 & 3.40 & 0.082 \\
\hline Maximum load (kg) & $\mathrm{CN}$ & & 0.06 & 90 & 230 & 0.003 \\
\hline Total & - & - & 1 & - & - & 0.632 \\
\hline
\end{tabular}

\section{Conclusions}

This paper proposes a knowledge service method that supports the intelligent design of products from the aspect of demands for intelligent design in the actual product design process. The proposed method can effectively solve the calculation problems and reasoning and decision-making problems of the intelligent product design activities, providing support to the whole intelligent design process by using design knowledge. A global similarity measurement method is proposed for the scheme reasoning. Compared with the existing methods for hybrid similarity measurement, the proposed method has distinct characteristics, which are given in the following:

(1) The proposed hybrid similarity measurement method includes five formats of attribute values: CS, $\mathrm{CN}, \mathrm{FN}, \mathrm{FL}$, and FI. In this method, the attribute similarity for each format of the attribute value is calculated.

(2) The proposed hybrid similarity measurement method integrates the fuzzy set similarity theory and traditional similarity measurement method. In addition, the derived similarity measurement equation for fuzzy attribute sets can solve the problems of complex and inaccurate fuzzy attribute retrieval in the traditional similarity measurement.

(3) The attribute level classification method proposed to assign weight coefficients is based on the analysis of product structure and performance indicators. The attributes are classified into primary matching and auxiliary matching levels according to the importance and decisiveness of product attribute type to case matching, which can reduce the time to find an optimal solution. The set of weight coefficients can be continuously and dynamically updated through case-based reasoning learning so that it is more in line with the actual design situation of the product.

(4) A global similarity measurement is performed by combining the hybrid similarity measurement and the level-based assignment of weight coefficients, which can improve solving the core problem of case retrieval in the CBR reasoning system. Through the design of CNC tool holder products, the effectiveness of the intelligent design system is verified. This paper presents an application of the hybrid similarity measure method in the field of mechanical product design, which is different from the previous work.

The future research work could include the following aspects:

(1) The intelligent service of design knowledge could be further improved regarding the design process. Problems such as design knowledge-assisted decision-making through knowledge reasoning could be solved. In addition, a dynamic relationship between design knowledge and the design process could be achieved.

(2) The proposed intelligent calculation method could be embedded in the CBR system. The object's characteristic attributes used for case matching cannot completely determine the reusability of the case, so various evaluation indicators need to be comprehensively considered, such as failure rate, structural simplicity, maintainability, safety, and economic cost. In future work, a scheme evaluation method that combines the gray correlation analysis 
and the entropy method could be used to obtain a similar optimal case as a target scheme.

(3) The proposed method could also be applied to case retrieval in a wider range of applications, such as medical diagnosis, emergency management, and engineering cost.

\section{Data Availability}

The data used to support the findings of this study are included within the article.

\section{Conflicts of Interest}

The authors declare that there are no conflicts of interest in this article.

\section{Acknowledgments}

This work was supported by the National Science and Technology Major Project under Grant no. 2013ZX04012032 and the Jinling Science and Engineering High-Level Talent Research Startup Project under Grant no. jit-b-201908.

\section{References}

[1] A. E. Maxwell, T. A. Warner, and F. Fang, "Implementation of machine-learning classification in remote sensing: an applied review," International Journal of Remote Sensing, vol. 39, no. 9, pp. 2784-2817, 2018.

[2] P. Wang, Y. Gong, H. Xie, Y. Liu, and A. Y. Nee, “Applying $\mathrm{CBR}$ to machine tool product configuration design oriented to customer requirements," Chinese Journal of Mechanical Engineering, vol. 30, no. 1, pp. 60-76, 2017.

[3] Y. Guo, J. Hu, and Y. Peng, "A CBR system for injection mould design based on ontology: a case study," ComputerAided Design, vol. 44, no. 6, pp. 496-508, 2012.

[4] S.-Y. Zhang, Y. Gu, X.-J. Liu, and J.-R. Tan, "A knowledge push technology based on applicable probability matching and multidimensional context driving," Frontiers of Information Technology \& Electronic Engineering, vol. 19, no. 2, pp. 235-245, 2018.

[5] M. R. Khosravani and S. Nasiri, "Injection molding manufacturing process: review of case-based reasoning applications," Journal of Intelligent Manufacturing, vol. 31, no. 4, pp. 847-864, 2020.

[6] X. Wei, H. Yuan, H. Wang, and Y. Chen, "Intelligent design for automotive interior trim structures based on knowledge rule-based reasoning," International Journal of Automotive Technology, vol. 21, no. 5, pp. 1149-1167, 2020.

[7] H. Zhang and L. Yu, "New distance measures between intuitionistic fuzzy sets and interval-valued fuzzy sets," Information Sciences, vol. 245, pp. 181-196, 2013.

[8] D. A. Sharaf-El-Deen, I. F. Moawad, and M. E. Khalifa, "A new hybrid case-based reasoning approach for medical diagnosis systems," Journal of Medical Systems, vol. 38, pp. 1-11, 2014.

[9] W. Qian, X. Peng, B. Chen, J. Mylopoulos, H. Wang, and W. Zhao, "Rationalism with a dose of empiricism: combining goal reasoning and case-based reasoning for self-adaptive software systems," Requirements Engineering, vol. 20, no. 3, pp. 233-252, 2015.
[10] D. Gu, C. Liang, and H. Zhao, "A case-based reasoning system based on weighted heterogeneous value distance metric for breast cancer diagnosis," Artificial Intelligence in Medicine, vol. 77, pp. 31-47, 2017.

[11] C. Tan, W. Yi, and X. Chen, "Hesitant fuzzy Hamacher aggregation operators for multicriteria decision making," Applied Soft Computing, vol. 26, pp. 325-349, 2015.

[12] Y. Song, X. Wang, W. Quan, and W. Huang, "A new approach to construct similarity measure for intuitionistic fuzzy sets," Soft Computing, vol. 23, no. 6, pp. 1985-1998, 2019.

[13] T. Mahmood, U. Ur Rehman, Z. Ali, and T. Mahmood, "Hybrid vector similarity measures based on complex hesitant fuzzy sets and their applications to pattern recognition and medical diagnosis," Journal of Intelligent and Fuzzy Systems, vol. 40, no. 1, pp. 625-646, 2021.

[14] K. Feng, D.-F. He, A.-J. Xu, and H.-B. Wang, "End temperature prediction of molten steel in $\mathrm{RH}$ based on case-based reasoning with optimized case base," Journal of Iron and Steel Research International, vol. 22, no. S1, pp. 68-74, 2015.

[15] M. J. Khan, H. Hayat, and I. Awan, "Hybrid case-base maintenance approach for modeling large scale case-based reasoning systems," Human-centric Computing and Information Sciences, vol. 9, pp. 1-25, 2019.

[16] C. Feng, N. D. Yang, W. M. Gui, and L. Zou, "Method for generating emergency alternative based on case-based reasoning," Control and Decision, vol. 31, no. 8, pp. 1526-1530, 2016.

[17] G. Xiong, J. Lan, H. Zhang, and T.-H. Ding, "The effect of attribute normalization factors in attribute distance weighted average," Automatic Control and Computer Sciences, vol. 51, no. 2, pp. 85-96, 2017.

[18] S. Ahmadvand and M. S. Pishvaee, "An efficient method for kidney allocation problem: a credibility-based fuzzy common weights data envelopment analysis approach," Health Care Management Science, vol. 21, no. 4, pp. 587-603, 2018.

[19] Y.-L. Li, C.-S. Ying, K.-S. Chin, H.-T. Yang, and J. Xu, “Thirdparty reverse logistics provider selection approach based on hybrid-information MCDM and cumulative prospect theory," Journal of Cleaner Production, vol. 195, no. 10, pp. 573-584, 2018.

[20] M. Lin, H. Wang, and Z. Xu, "TODIM-based multi-criteria decision-making method with hesitant fuzzy linguistic term sets," Artificial Intelligence Review, vol. 53, no. 5, pp. 3647-3671, 2020.

[21] Z. S. Chen, X. Zhang, W. Pedrycz, X. J. Wang, and M. J. Skibniewski, "Bid evaluation in civil construction under uncertainty: a two-stage LSP-ELECTRE III-based approach," Engineering Applications of Artificial Intelligence, vol. 94, pp. 1-18, 2020.

[22] P. Sun, S. Chen, and Y. Zhi, "Multiple attribute variable weight fuzzy decision-making based on optimistic coefficient method," International Journal of Fuzzy Systems, vol. 23, no. 2, pp. 573-583, 2021.

[23] G. X. Zhao, Z. D. Yan, and M. Peter, "Knowledge-driven intelligent quality problem-solving system in the automotive industry," Advanced Engineering Informatics, vol. 38, pp. 441-457, 2018.

[24] L. Gao, K. Dai, L. Gao, and T. Jin, "Expert knowledge recommendation systems based on conceptual similarity and space mapping," Expert Systems with Applications, vol. 136, pp. 242-251, 2019.

[25] C. K. H. Lee, "A knowledge-based product development system in the chemical industry," Journal of Intelligent Manufacturing, vol. 30, no. 3, pp. 1371-1386, 2019. 
[26] C. Richard, J. C. V. Wim, J. L. Michel, and T. H. van der Laan, "A multi-disciplinary implementation methodology for knowledge based engineering: knomad," Expert Systems with Applications, vol. 37, no. 11, pp. 7336-7350, 2010.

[27] S. Ahmed-Kristensen and G. Vianello, "A model for reusing service knowledge based on an empirical case," Research in Engineering Design, vol. 26, no. 1, pp. 57-76, 2015.

[28] Y. Cui, L. Zhang, Y. Hou, and G. Tian, "Design of intelligent home pension service platform based on machine learning and wireless sensor network," Journal of Intelligent and Fuzzy Systems, vol. 40, no. 2, pp. 2529-2540, 2021.

[29] M. Moravik, M. Schmid, and N. Burch, "Deepstack: expertlevel artificial intelligence in heads-up no-limit poker," Science, vol. 356, pp. 508-513, 2017.

[30] S. Lutz, "Empiricism and intelligent design II: analyzing intelligent design," Erkenntnis, vol. 78, no. 3, pp. 681-698, 2013.

[31] D. Li, W. Zeng, and J. Li, "New distance and similarity measures on hesitant fuzzy sets and their applications in multiple criteria decision making," Engineering Applications of Artificial Intelligence, vol. 40, pp. 11-16, 2015.

[32] L. R. Gianfranco, "Knowledge based engineering: between AI and CAD. Review of a language based technology to support engineer-ing design," Advanced Engineering Informatics, vol. 26, no. 2, pp. 159-179, 2012.

[33] Z. Li, X. Zhou, W. M. Wang, G. Huang, Z. Tian, and S. Huang, "An ontology-based product design framework for manufacturability verification and knowledge reuse," International Journal of Advanced Manufacturing Technology, vol. 99, no. 9-12, pp. 2121-2135, 2018.

[34] S. Ren, Y. W. Zhao, and H. H. Hong, "A retrieval method for similar cases with multiple attributes in low-carbon design," Journal of Mechanical Engineering, vol. 55, no. 1, pp. 149-159, 2019.

[35] M. Nicolás and O. A. Manuel, "Functional degrees of inclusion and similarity between L-fuzzy sets," Fuzzy Sets and Systems, vol. 390, pp. 1-22, 2020.

[36] S. Abir and E. Zied, "Dynamic maintenance case base using knowledge discovery techniques for case based reasoning systems," Theoretical Computer Science, vol. 817, pp. 24-32, 2020.

[37] Z. Wu, L. He, Y. Wang, M. Goh, and X. Ming, "Knowledge recommendation for product development using integrated rough set-information entropy correction," Journal of Intelligent Manufacturing, vol. 31, no. 6, pp. 1559-1578, 2020.

[38] D. Wang, Y. H. Yih, and M. Ventresca, "Improving neighborbased collaborative filtering by using a hybrid similarity measurement," Expert Systems with Applications, vol. 160, pp. 1-17, 2020.

[39] K. Ullah, H. Garg, T. Mahmood, N. Jan, and Z. Ali, "Correlation coefficients for T-spherical fuzzy sets and their applications in clustering and multi-attribute decision making," Soft Computing, vol. 24, no. 3, pp. 1647-1659, 2020.

[40] H.-B. Yan and T. Ma, "A group decision-making approach to uncertain quality function deployment based on fuzzy preference relation and fuzzy majority," European Journal of Operational Research, vol. 241, no. 3, pp. 815-829, 2015.

[41] P. Liu, Z. Ali, and T. Mahmood, "A method to multi-attribute group decision-making problem with complex q-rung orthopair linguistic information based on heronian mean operators," International Journal of Computational Intelligence Systems, vol. 12, no. 2, pp. 1465-1496, 2019.

[42] B. Zhu and Z. Xu, "A fuzzy linear programming method for group decision making with additive reciprocal fuzzy preference relations," Fuzzy Sets and Systems, vol. 246, pp. 19-33, 2014.

[43] A. Inusah and T. Esko, "A paraconsistent many-valued similarity method for multi-attribute decision making," Fuzzy Sets and Systems, vol. 7, pp. 1-25, 2020. 